\title{
Errors in Pointing are Due to Approximations in Sensorimotor Transformations
}

\author{
JOHN F. SOECHTING AND MARTHA FLANDERS \\ Department of Physiology, University of Minnesota Minneapolis, Minnesota 55455
}

\section{SUMMARY AND CONCLUSIONS}

1. We define an extrinsic frame of reference to represent the location of a point in extrapersonal space relative to a human subject's shoulder, and we define an intrinsic frame of reference to represent the orientation of the arm and forearm.

2 . We examined the relations between coordinates in the extrinsic and intrinsic frames of reference under two experimental conditions: when subjects made inaccurate movements by pointing to virtual targets in the dark and when they made accurate movements by pointing to actual targets in the light.

3. When subjects made inaccurate movements, there was a close-to-linear relationship between the orientation angles of the arm (intrinsic coordinates) at its final position and the extrinsic coordinates of the target. When they made accurate movements, these relationships were more nonlinear.

4. Specifically, arm and forearm elevations depended principally on target distance and elevation, whereas the two yaw angles depended mainly on the target's azimuth.

5 . We propose that errors in pointing occur because subjects implement a linear approximation to the transformation from extrinsic to intrinsic coordinates and that this transformation is one step in the process of transforming a visually derived representation of target location into an appropriate pattern of muscle activity.

\section{INTRODUCTION}

In the preceding paper (Soechting and Flanders 1989) we showed that subjects made large and consistent errors when they pointed with their arm to remembered target locations in three-dimensional space. Since we also showed that the visually derived representation of target location was not in error, we concluded that the errors occurred somewhere in the process of transforming this visually derived representation into the pattern of muscle activity that is appropriate for attaining the target.

There appears to be a reasonably accurate internal representation of target location in a body-centered frame of reference (Andersen 1987; Sparks et al. 1986, 1987). We refer to the coordinates of this representation as extrinsic coordinates since they describe the location of an object extrinsic to the subject. There exists also an internal representation of the orientation of the upper arm and of the forearm (Soechting and Ross 1984). These orientation angles are taken to constitute an intrinsic coordinate system since they describe the orientation of body segments relative to each other. From these intrinsic coordinates the position of the hand in space can be calculated. The mathematically exact relationships between these intrinsic and extrinsic coordinates are highly nonlinear (Soechting et al. 1986a,b).

We begin with the following hypothesis which is consistent with results presented in the preceding paper (Soechting and Flanders 1989): During pointing movements, a sensorimotor transformation between these two internal representations is effected and is utilized to guide the finger to the vicinity of the target. Furthermore, the transformation which is utilized involves approximations to the mathematically exact solution (Greene 1972; Nashner and McCollum 1985). These approximations can lead to appreciable movement errors when visually derived information about the location of the target and/or the hand is unavailable for error correction.

In this paper we will present evidence in favor of this hypothesis. When subjects pointed (inaccurately) to remembered target locations, the relations between intrinsic and extrinsic coordinates were simple, being close to linear. Instead when the movements were accurate, these relations were more complicated and nonlinear.

\section{METHODS}

In this paper we will examine in more detail the results of two of the motor tasks described in the preceding paper (Soechting and Flanders 1989). In the first task, subjects were presented with a target, the target was then removed, the room lights were extinguished, and the subjects pointed to the remembered location of the target (Virtual Target, Dark). As shown in the preceding paper, the subjects sometimes made appreciable errors in this experimental condition, i.e., their finger could be as much as 15 $\mathrm{cm}$ away from the target position at the end of the movement. In the second task, subjects pointed to a target which remained in place and then were asked to reproduce the movement after the target had been removed (Reproduce Active Movement). By definition, the initial movement to the target was an "Accurate Movement."

In each task, the final position of the shoulder, elbow, wrist, and finger was recorded ultrasonically, as was the target location. (To be precise, the position of the tip of the stylus grasped by the subject was recorded. We shall refer to this point as the position of the finger.) For the second task, we will consider in this paper only the posture of the arm at the end of the initial movement, i.e., when the location of the finger and of the target coincided. Four subjects participated in each of the two tasks, each subject being presented with 60 to 100 different targets distributed randomly in space. 


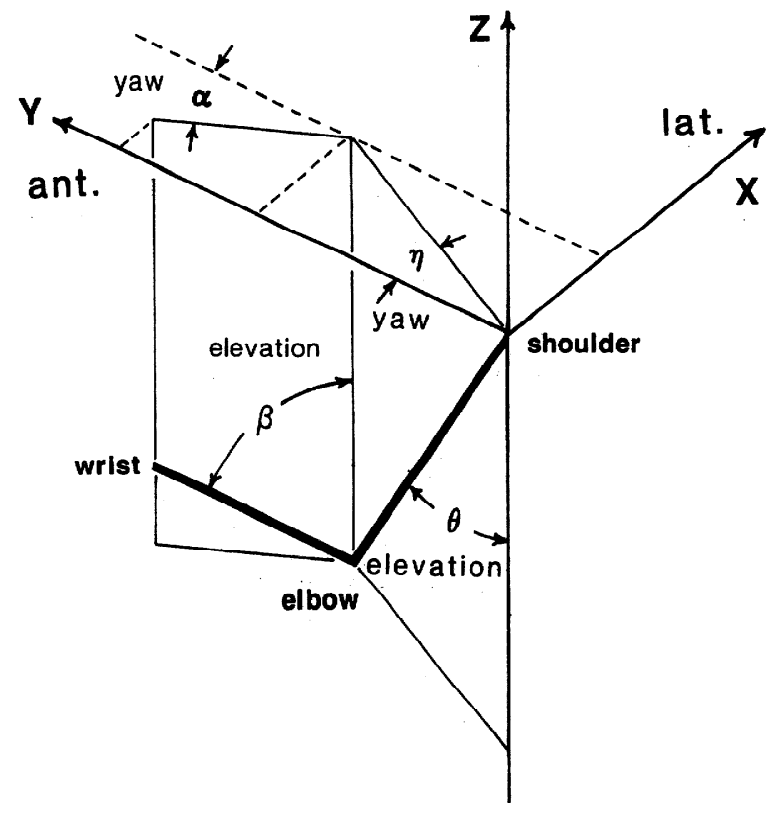

FIG. 1. Orientation angles of the arm and forearm. The angles $\theta$ and $\eta$ define the elevation and the yaw of the upper arm and $\beta$ and $\alpha$ represent the elevation and yaw of the forearm. Elevation is defined as the angle between the limb segment and the vertical axis and is measured in a vertical plane. Yaw is the angle between the limb segment and the anterior direction, measured in the horizontal plane.

\section{Orientation angles}

From the measured position of the shoulder, elbow, and wrist, we computed the orientation angles of the arm and forearm. These angles are defined in Fig. 1. While they could have been defined differently, we chose this particular set of coordinates since it had been shown previously to constitute a preferred coordinate system for the recognition of the orientation of the arm in space (Soechting et al. 1982, 1984; Worringham et al. 1987). Two angles define the orientation of the upper arm: yaw $(\eta)$ and elevation $(\theta)$, and two more define the orientation of the forearm: yaw $(\alpha)$ and elevation $(\beta)$. The elevations $(\theta$ and $\beta)$ define the angle between each limb segment and the vertical axis and are measured in a vertical plane. The yaw angles ( $\eta$ and $\alpha$ ) define the angle between each of the limb segments and the anterior direction, measured in the horizontal plane.

These orientation angles $(\eta, \theta, \alpha, \beta)$ constitute the intrinsic coordinates. For the extrinsic coordinates which represent the position of the target in space, we use the spherical coordinate system with origin at the shoulder that was defined in the preceding paper (Soechting and Flanders 1989). In this coordinate system $R$ denotes the radial distance, $\chi$ the azimuth, and $\psi$ the elevation. We chose spherical coordinates rather than Cartesian or cylindrical because, according to the criteria described in the preceding paper (Soechting and Flanders 1989), the former lead to a more compact representation of the relations between target and finger location.

Both the intrinsic and extrinsic coordinates are most easily defined with reference to the Cartesian coordinates illustrated in Fig. 1. In this coordinate system the position of the wrist is given by

$$
\begin{aligned}
& x_{\mathrm{w}}=l_{\mathrm{a}} \sin \theta \sin \eta+l_{\mathrm{f}} \sin \beta \sin \alpha \\
& y_{\mathrm{w}}=l_{\mathrm{a}} \sin \theta \cos \eta+l_{\mathrm{f}} \sin \beta \cos \alpha \\
& z_{\mathrm{w}}=-l_{\mathrm{a}} \cos \theta+l_{\mathrm{f}} \cos \beta
\end{aligned}
$$

where $l_{\mathrm{a}}$ and $l_{\mathrm{f}}$ are the lengths of the arm and forearm, respectively. The position of the target is given by

$$
\begin{aligned}
R^{2} & =x^{2}+y^{2}+z^{2} \\
\tan \chi & =x / y \\
\tan \psi & =z / \sqrt{\left(x^{2}+y^{2}\right)}
\end{aligned}
$$

Two points should be noted regarding the relation between the intrinsic and extrinsic coordinates defined in Eq. 1. First, these relations are obviously nonlinear. Second, for a given position of the wrist there does not correspond a unique orientation of the limb: three parameters $\left(x_{\mathrm{w}}, y_{\mathrm{w}}, z_{\mathrm{w}}\right)$ are required to define the former, whereas four $(\eta, \theta, \alpha, \beta)$ are needed to specify the latter. Both points would be equally valid in any other coordinate system used to define the position of the wrist.

\section{Multivariate regression analysis}

The aim of the analysis presented in this paper was to understand the nature of the transformation between extrinsic and intrinsic coordinates in the two motor tasks. To this end we needed to characterize quantitatively how each of the intrinsic parameters depend on each of the extrinsic variables. We therefore performed a regression analysis between each of the four orientation angles and the parameters defining target location, using procedures similar to those described in the preceding paper (Soechting and Flanders 1989). We investigated linear, quadratic, and cubic polynomial models. For each model we retained only those terms which were significant at the $95 \%$ confidence level (Johnson and Wichern 1982).

For example, in the case of a linear model

$$
\theta=\mathrm{a}_{0}+\mathrm{a}_{1} R+\mathrm{a}_{2} \chi+\mathrm{a}_{3} \psi+\epsilon
$$

where $\theta$ is the elevation of the upper arm, and $R, \chi$, and $\psi$ are the radial distance, azimuth, and elevation of the target, respectively. The variable $\epsilon$ represents the error, i.e., the difference between the measured value of $\theta$ in each trial and the value predicted by the linear model. The extent to which linear or cubic models could fit the data (using only those terms which were significant) was ascertained by computing the variance of the error $\epsilon$. If $\epsilon_{\mathrm{c}}{ }^{2}$ is the variance of the cubic model and $\epsilon_{1}^{2}$ the variance associated with the linear model, the extent to which the former gives a better fit to the data is given by

$$
\Delta=\sqrt{\epsilon_{1}^{2}-\epsilon_{\mathrm{c}}{ }^{2}}
$$

For each model, we also computed the multiple correlation coefficient $r$.

\section{RESULTS}

The results of our analysis of the relationship between intrinsic variables (orientation angles of the arm) and the extrinsic variables (target location) can be summarized as follows: When subjects made movements which could be inaccurate (Virtual Target, Dark) this relationship was close-to-linear. When they made accurate movements to targets, the final orientation of the arm was related to target location in a manner which was significantly more nonlinear.

These results are illustrated in Figs. 2-9 and statistical details are given in Tables $1-4$. Since there was some variability in the behavior among subjects we provide both illustrative data for all subjects for one experimental condition (Figs. 2, 3, 6, and 7), and the results of the analysis 

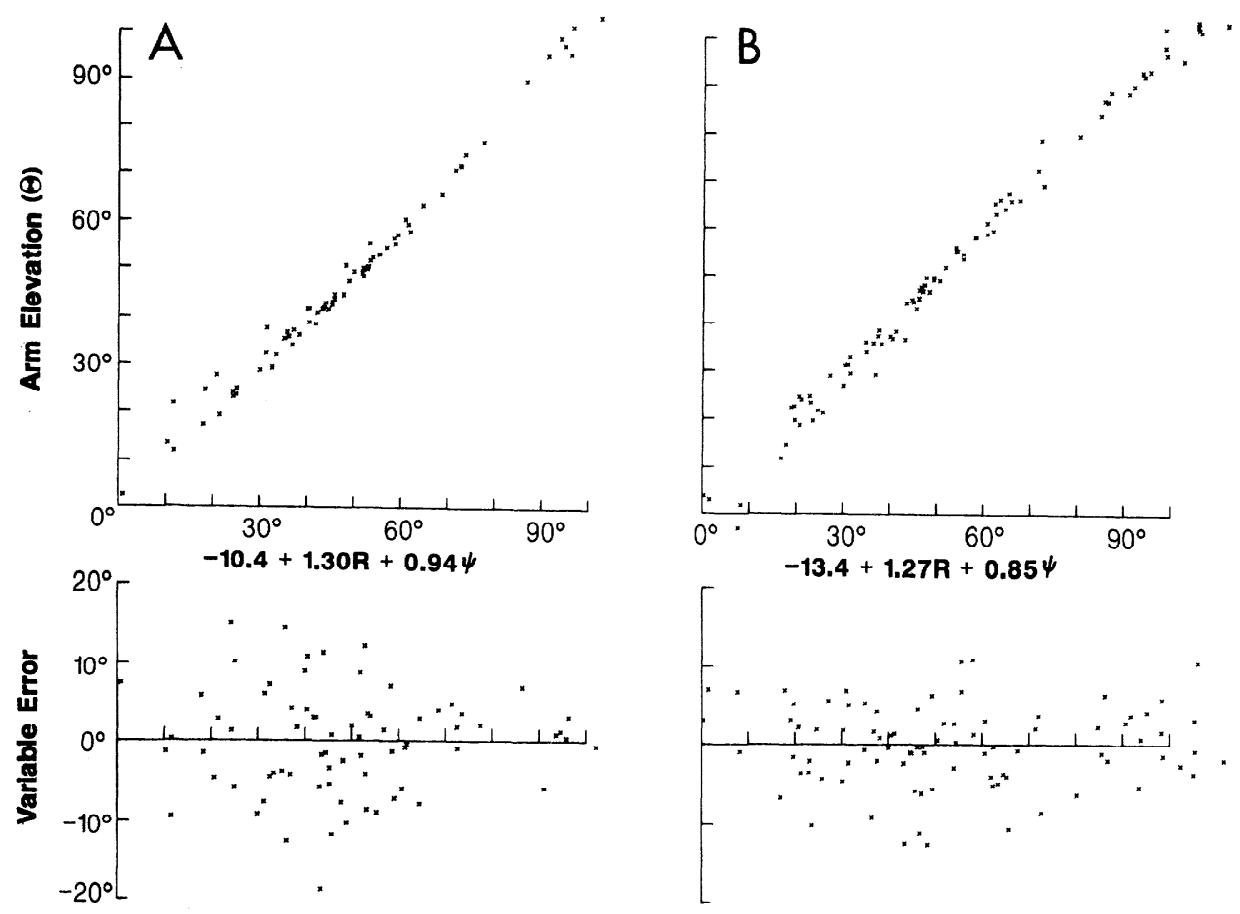

FIG. 2. Dependence of arm elevation on target distance $(R)$ and elevation $(\psi)$. The data in each panel $(A-D)$ depict results from 1 of the 4 subjects (A-D, Table 1) who pointed to a virtual target in the dark. The horizontal axis in each panel is the linear combination of target parameters which gave the best fit to the data, whereas the vertical axis represents the value of $\theta$ predicted by the nonlinear model. Variable error in $A$ and $B$ is the difference between the experimental data and the model's prediction for each trial.
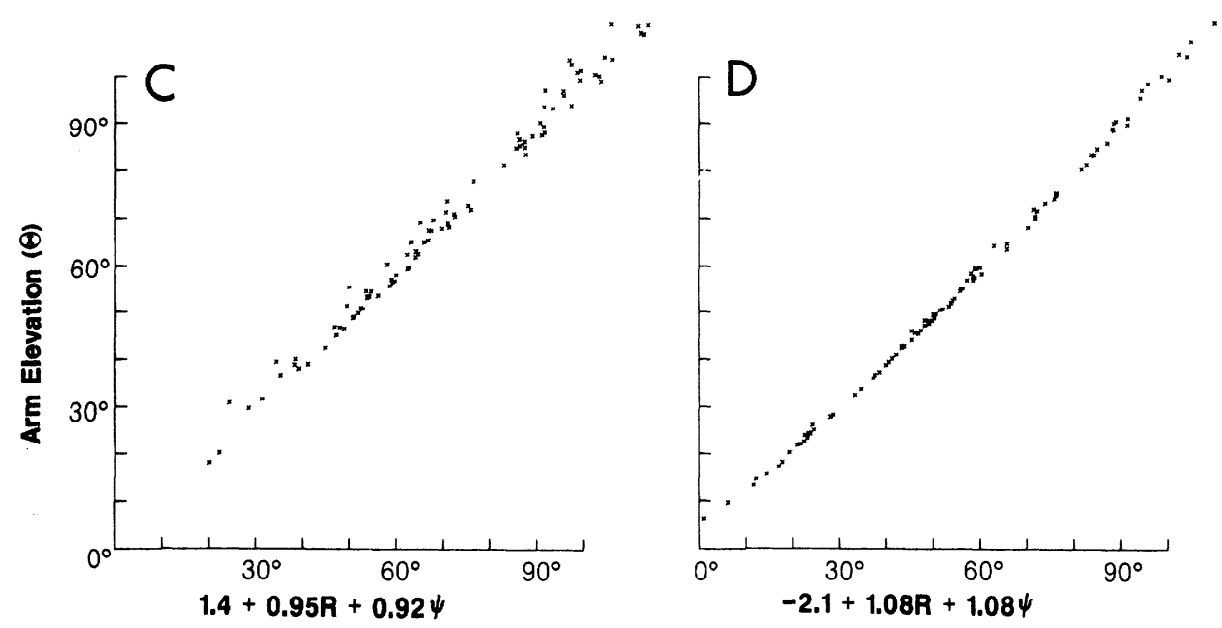

when data from all four subjects are combined (Figs. 4, 5, 8, and 9).

We begin by considering the dependence of arm elevation $(\theta)$ and forearm elevation $(\beta)$ on target location. Figure 2 presents the results regarding arm elevation for the four subjects who pointed to a virtual target in the dark. The horizontal axis in each panel is the linear combination of the extrinsic parameters which gave the best fit to arm elevation $(\theta)$ for each subject, whereas the vertical axis represents the value of $\theta$ predicted by the cubic polynomial model. Each data point presents the results from one trial. The scatter of the data points about a straight line with a slope of 1.0 gives an indication of the extent to which arm elevation depends in a nonlinear manner on target location, i.e., of the extent to which quadratic and cubic terms contributed to the fit of the data. For the first two subjects

( $A$ and $B$ ), we show also the difference between the value $\theta$ predicted by the cubic polynomial and the measured elevation of the arm, i.e., the error $\epsilon$.

In all four subjects arm elevation $\theta$ depended linearly only on the radial distance of the target from the shoulder $(R)$ and on the target's elevation $(\psi)$. According to the linear model, the dependence of $\theta$ on target azimuth $(\chi)$ was insignificant. The further away the target was (larger values of $R$ ), the greater was arm elevation. Similarly, the higher the target was (larger values of $\psi$ ), the greater was $\theta$. It is evident in Fig. 2 that the scatter of the data points about a straight line is modest in all four subjects. Accordingly the nonlinear (cubic) model did not produce a large improvement in the goodness of the fit. Statistical measures are given in Table 1 for each subject. The values of the multiple correlation coefficient $\left(r^{2}\right)$ for the linear model were highly 

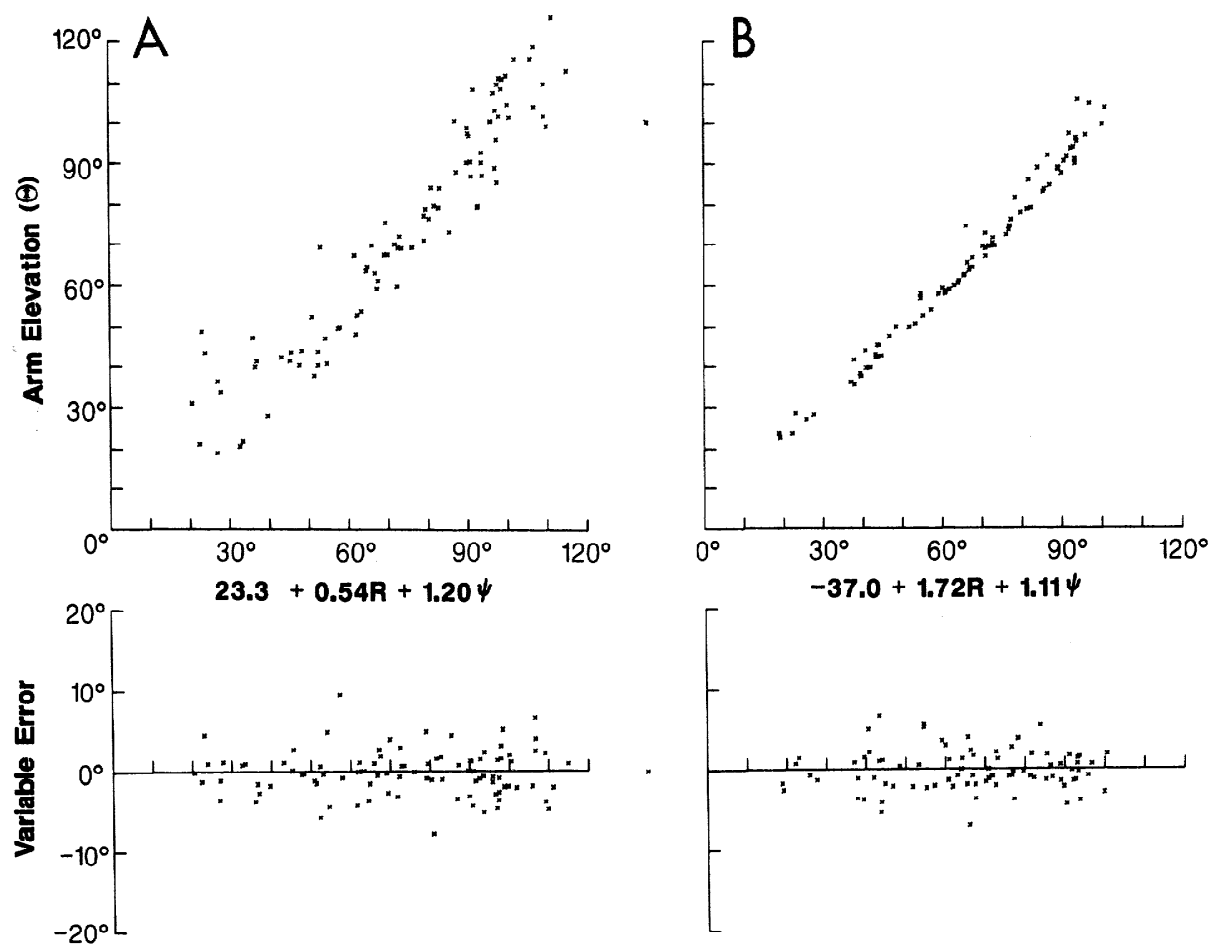

FIG. 3. Dependence of arm elevation on target distance $(R)$ and elevation $(\psi)$. The data in each panel $(A-D)$ depict results from 1 of the 4 subjects who made accurate movements to targets $(E-H$, respectively, in Table 1$)$. Format of the plots is the same as in Fig. 2.
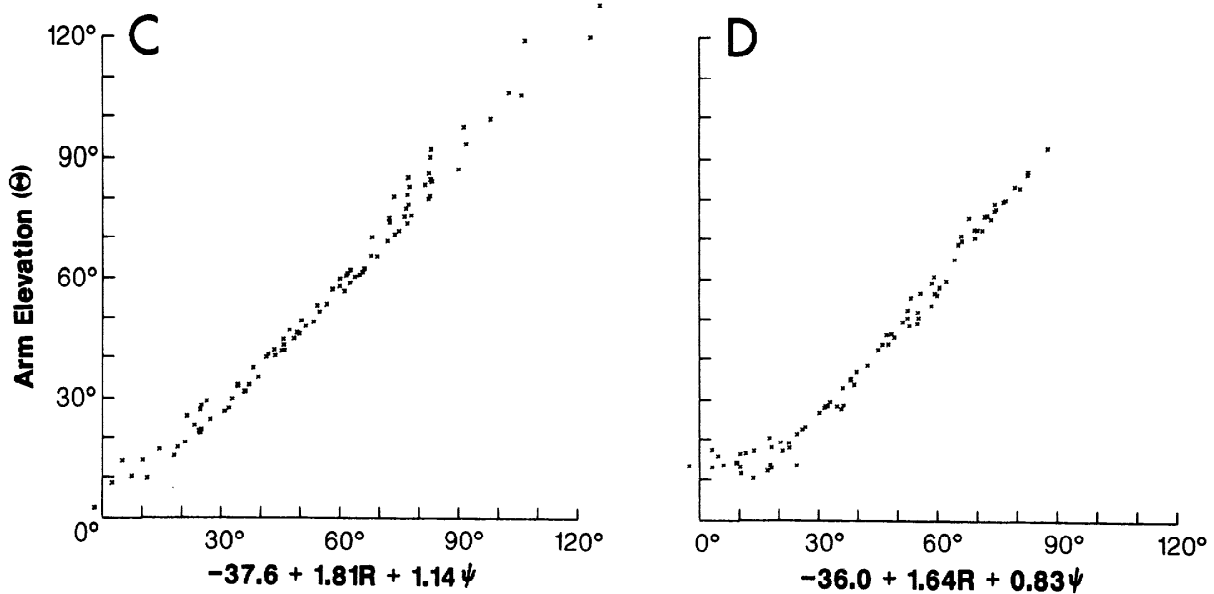

significant, ranging from 0.906 to 0.965 . The extent to which the nonlinear model reduced the error is given by $\Delta$ (see $E q$. 4). This value ranged from 1.2 to $2.9^{\circ}$.

The root mean square (RMS) error of the cubic model $\left(\epsilon_{c}\right)$, which ranged from 4.0 to $6.6^{\circ}$ in the four subjects, can potentially have a number of sources. One possibility is that the model was inadequate, i.e., that terms of order greater than three were needed. This is unlikely since only three to six coefficients (out of a total of 20 possible coefficients) were significant in fitting the data in Fig. 2. A second source is measurement error, e.g., due to slippage of the transducers with respect to the skeletal frame. This error is unlikely to exceed $1^{\circ}$. The major source of error is most likely due to the variability in pointing described in the preceding paper (Soechting and Flanders 1989). In this context, it may be noted that the RMS error in $\epsilon\left(\epsilon_{\mathrm{c}}\right)$ re- ported here is comparable to errors obtained when subjects were asked to match upper arm elevations with their right and left arms (Soechting and Ross 1984) and more generally with the error in joint angle matching tasks (Goodwin et al. 1972; Horch et al. 1975; Laszlo and Bairstow 1983; Paillard 1973).

As was stated before and as is evident in Fig. 3, nonlinear terms in the dependence of arm elevation $(\theta)$ on the parameters describing target location are more prominent when subjects made accurate movements to the target. The results from the four subjects who performed this task are shown in Fig. 3 in the same format used in Fig. 2. The abscissa in each panel shows the combinations of parameters in the linear model which gave the best fit to the data. As in Fig. 2, arm elevation $(\theta)$ depended most strongly on radial distance $(R)$ and target elevation $(\psi)$. However, the 
scatter in the data points about a straight line with a slope of 1.0 can be much greater than in Fig. 2 (see Fig. $3 A$ ) or the data can deviate significantly from a straight line (Fig. $3 D$ ). The extent to which the nonlinear model improved the fit to the data $(\Delta)$ ranged from 2.8 to $9.0^{\circ}$ (see Table 1). Only for one subject (Fig. $3 B$ ) did this value fall within the range of values found in the first experimental condition $(1.2$ to $2.9^{\circ}$ ).

Finally, the RMS error of the cubic model $\left(\epsilon_{\mathrm{c}}\right)$ was quite small, ranging from 2.2 to $2.8^{\circ}$. Note that in this experimental condition there was, by design, no error in the final position of the finger. The error which remains represents in part measurement error and in part the variability in arm orientation corresponding to a unique position of the finger. (Recall that the arm has four degrees of freedom, therefore there is more than one posture of the arm possible for any one position of the wrist.) Both of these sources of variability are much smaller than the variability in the data for the first experimental condition (RMS values of $\sim 5^{\circ}$ ).
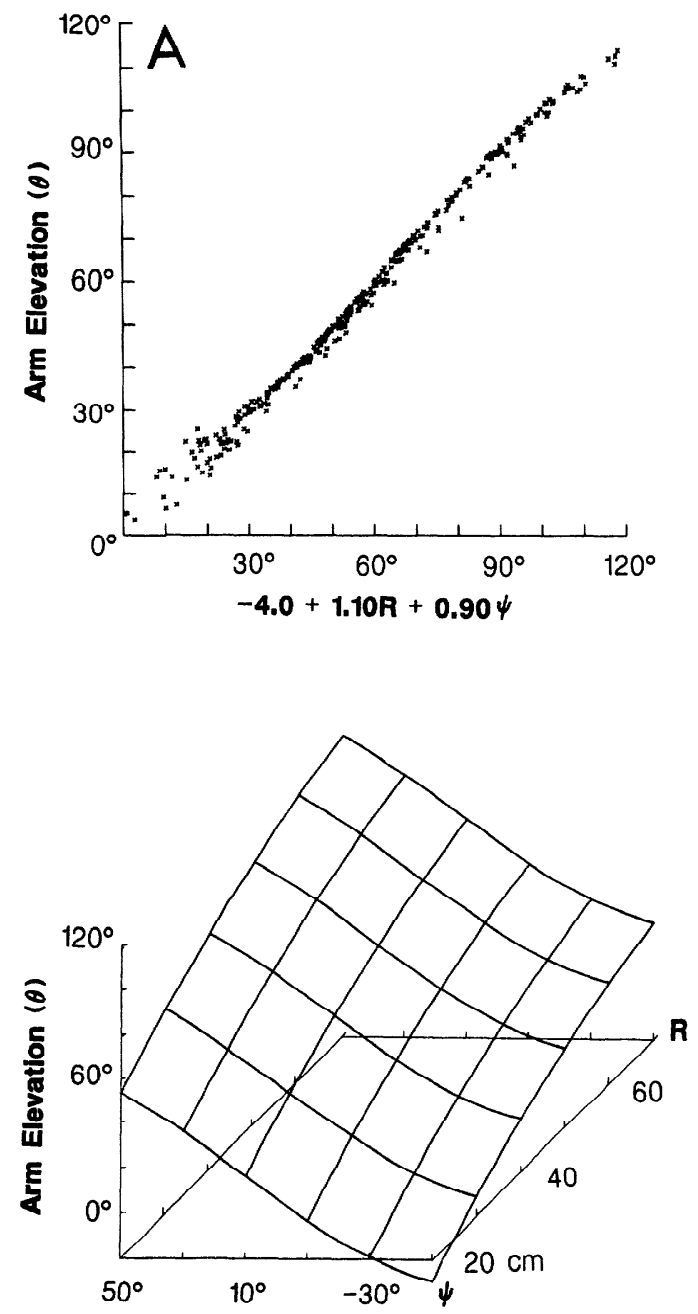

Figure 4 presents a comparison of the results between the two experimental conditions. In this figure, and in the other figures presented in this format (Figs. 5, 8, and 9), we focus on the aspects of the behavior common to the four subjects in each experimental condition by pooling the data. The plots in Fig. 4 (top) show the combined data from the subjects in the same format as in Figs. 2 and 3: the angular elevation of the arm $(\theta)$ predicted for movements to a virtual target in the dark (Fig. $4 A$ ) and for movements to an actual target in the light (Fig. $4 B$ ).

In the plots in Fig. 4 (bottom) we show the manner in which arm elevation depends on radial distance $(R)$, elevation $(\psi)$, and azimuth $(\chi)$ of the target, using a three-dimensional plot. These plots were generated by using the cubic polynomial in $R, \chi$, and $\psi$, which gave the best fit to the data and whose coefficients were all significant. The range of values for $R(20-70 \mathrm{~cm})$ and for $\chi$ and $\psi(-50$ to $50^{\circ}$ ) encompasses the space in which targets were located and are measured relative to the shoulder.
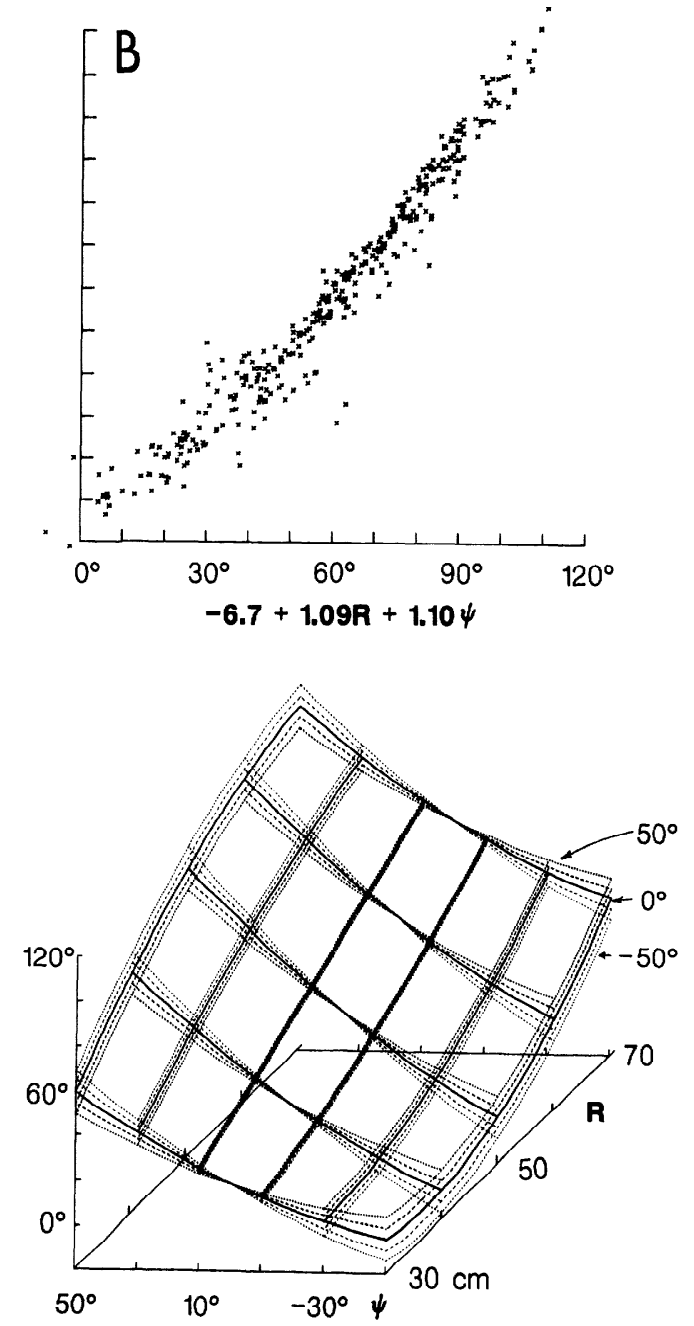

FIG. 4. Relationship between arm elevation $(\theta)$ and extrinsic parameters defining target location when subjects pointed to virtual targets $(A)$ and when they made accurate movements $(B) . A$ and $B$ : top panels depict pooled data from 4 subjects each. The panels below show in a 3-dimensional perspective plot the manner in which arm elevation depended on radial distance $(R)$, elevation $(\psi)$, and azimuth $(\chi)$ of the target as predicted by a cubic polynomial. This polynomial depended on $\chi$ in $B$ but not in $A$. Each surface in $B$ denoted by a different line symbol represents the predicted value of $\theta$ at one constant value of $\chi . \chi$ ranged from -50 to $50^{\circ}$ and the model's predictions are plotted in increments of $25^{\circ}$. 
TABLE 1. Upper arm elevation, $\theta$

\begin{tabular}{|c|c|c|c|c|c|c|}
\hline \multirow[b]{2}{*}{ Subject } & \multirow[b]{2}{*}{ Linear Model } & \multicolumn{2}{|c|}{ Error } & \multirow[b]{2}{*}{$\Delta$} & \multicolumn{2}{|c|}{$r^{2}$} \\
\hline & & $\epsilon_{1}$ & $\epsilon_{\mathrm{c}}$ & & Linear & Cubic \\
\hline \multicolumn{7}{|c|}{ Virtual target (dark) } \\
\hline $\begin{array}{l}A \\
B \\
C \\
D\end{array}$ & $\begin{array}{r}-10.4+1.30 R+0.94 \psi \\
-13.4+1.27 R+0.85 \psi \\
1.4+0.95 R+0.92 \psi \\
-2.1+1.08 R+1.08 \psi\end{array}$ & $\begin{array}{l}7.10 \\
5.83 \\
4.49 \\
5.68\end{array}$ & $\begin{array}{l}6.62 \\
5.06 \\
4.03 \\
5.54\end{array}$ & $\begin{array}{l}2.59 \\
2.89 \\
1.98 \\
1.23\end{array}$ & $\begin{array}{l}0.906 \\
0.958 \\
0.965 \\
0.955\end{array}$ & $\begin{array}{l}0.918 \\
0.968 \\
0.971 \\
0.957\end{array}$ \\
\hline Combined & $-4.0+1.10 R+0.90 \psi$ & 6.81 & 6.48 & 2.08 & 0.936 & 0.942 \\
\hline \multicolumn{7}{|c|}{ Accurate movement } \\
\hline $\begin{array}{l}E \\
F \\
G \\
H\end{array}$ & $\begin{array}{r}23.3+0.54 R+1.20 \psi \\
-37.0+1.72 R+1.11 \psi \\
-37.6+1.81 R+1.14 \psi \\
-36.0+1.64 R+0.83 \psi\end{array}$ & $\begin{array}{l}9.46 \\
3.72 \\
4.82 \\
5.34\end{array}$ & $\begin{array}{l}2.82 \\
2.48 \\
2.45 \\
2.19\end{array}$ & $\begin{array}{l}9.03 \\
2.77 \\
4.14 \\
4.87\end{array}$ & $\begin{array}{l}0.884 \\
0.970 \\
0.972 \\
0.953\end{array}$ & $\begin{array}{l}0.990 \\
0.987 \\
0.993 \\
0.992\end{array}$ \\
\hline Combined & $-6.7+1.09 R+1.10 \psi$ & 9.95 & 5.15 & 8.51 & 0.877 & 0.967 \\
\hline
\end{tabular}
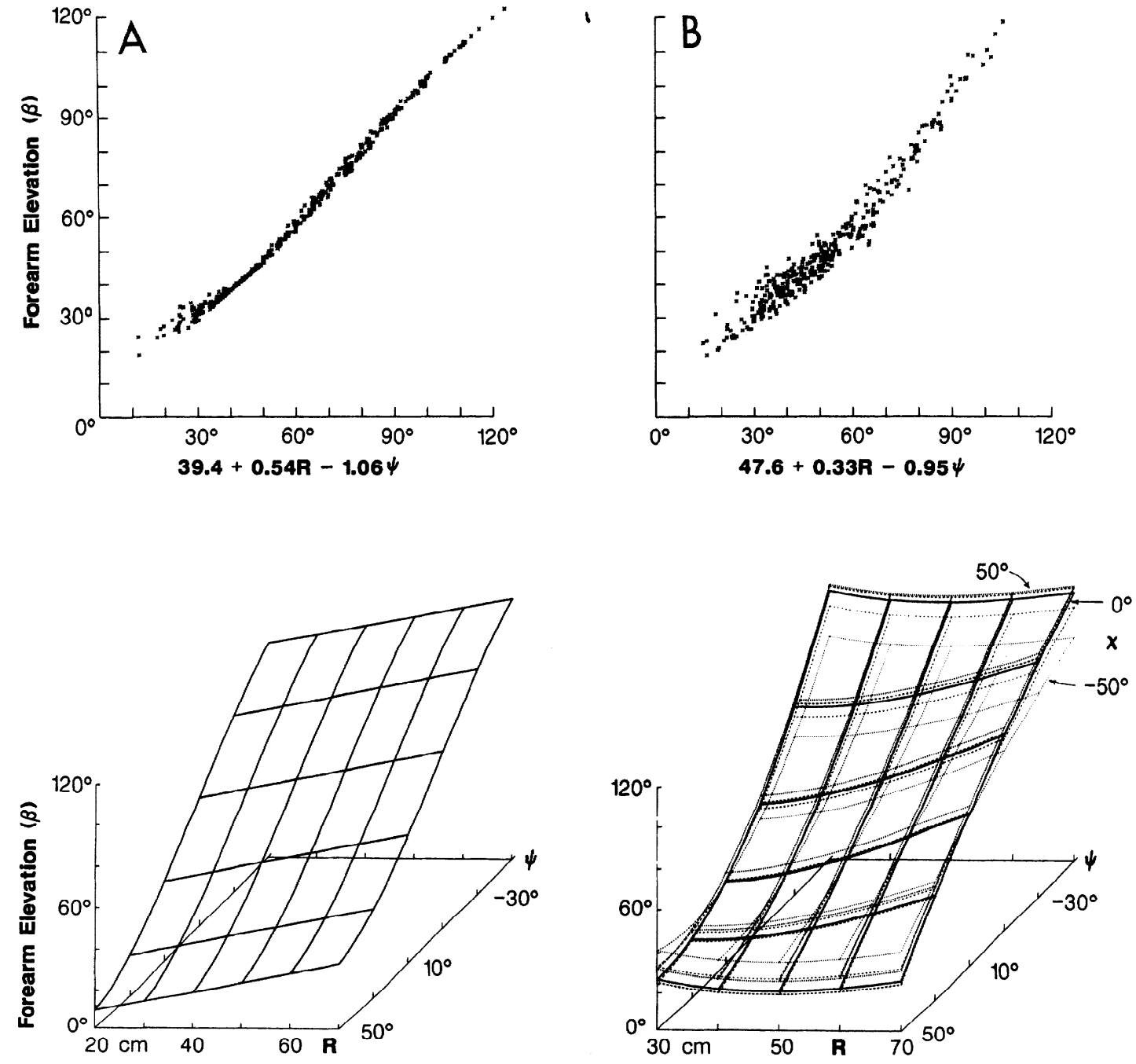

FIG. 5. Relationship between forearm elevation $(\beta)$ and extrinsic parameters when subjects pointed to virtual targets $(A)$ and when subjects made accurate movements $(B)$. The data are plotted in the same format as in Fig. 4. 
TABLE 2. Forearm elevation, $\beta$

\begin{tabular}{|c|c|c|c|c|c|c|}
\hline \multirow[b]{2}{*}{ Subject } & \multirow[b]{2}{*}{ Linear Model } & \multicolumn{2}{|c|}{ Error } & \multirow[b]{2}{*}{$\Delta$} & \multicolumn{2}{|c|}{$r^{2}$} \\
\hline & & $\epsilon_{1}$ & $\epsilon_{\mathrm{c}}$ & & Linear & Cubic \\
\hline \multicolumn{7}{|c|}{ Virtual target (dark) } \\
\hline $\begin{array}{l}A \\
B \\
C \\
D\end{array}$ & $\begin{array}{l}40.4+0.75 R-1.00 \psi \\
44.7+0.63 R-0.99 \psi \\
27.6+0.63 R-1.00 \psi \\
20.3+0.66 R-0.95 \psi\end{array}$ & $\begin{array}{l}6.25 \\
6.54 \\
4.54 \\
7.11\end{array}$ & $\begin{array}{l}5.53 \\
6.28 \\
3.29 \\
6.22\end{array}$ & $\begin{array}{l}2.91 \\
1.82 \\
3.13 \\
3.45\end{array}$ & $\begin{array}{l}0.929 \\
0.938 \\
0.958 \\
0.892\end{array}$ & $\begin{array}{l}0.944 \\
0.943 \\
0.978 \\
0.917\end{array}$ \\
\hline Combined & $39.4+0.54 R-1.06 \psi$ & 12.42 & 12.01 & 2.39 & 0.804 & 0.812 \\
\hline \multicolumn{7}{|c|}{ Accurate movement } \\
\hline $\begin{array}{l}E \\
F \\
G \\
H\end{array}$ & $\begin{array}{l}55.7+0.10 R-0.82 \psi \\
37.5+0.58 R-1.07 \psi \\
13.3+0.99 R-0.96 \psi \\
37.9+0.69 R-0.98 \psi\end{array}$ & $\begin{array}{l}7.08 \\
3.18 \\
6.76 \\
3.41\end{array}$ & $\begin{array}{l}2.48 \\
2.33 \\
3.03 \\
2.13\end{array}$ & $\begin{array}{l}6.64 \\
2.16 \\
6.04 \\
2.66\end{array}$ & $\begin{array}{l}0.849 \\
0.971 \\
0.903 \\
0.976\end{array}$ & $\begin{array}{l}0.982 \\
0.984 \\
0.981 \\
0.991\end{array}$ \\
\hline Combined & $47.6+0.33 R-0.95 \psi$ & 7.79 & 5.52 & 5.50 & 0.859 & 0.929 \\
\hline
\end{tabular}
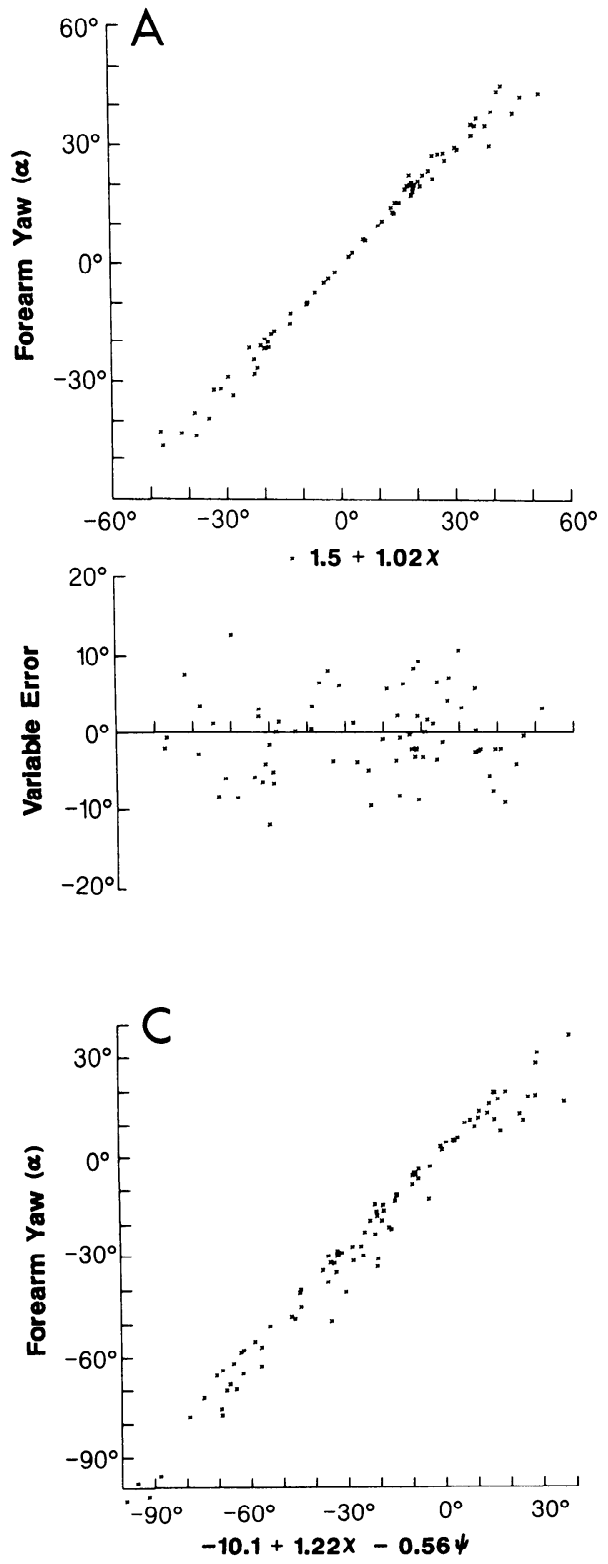
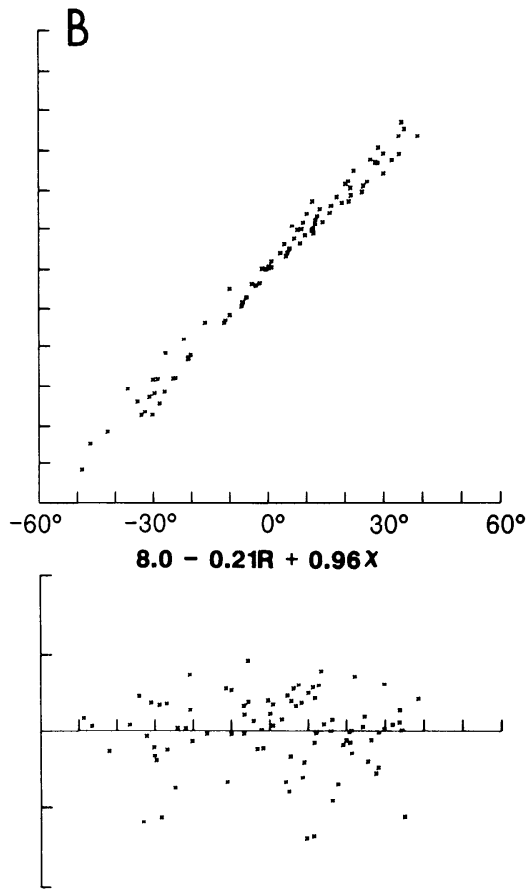

FIG. 6. Dependence of forearm yaw $(\alpha)$ on extrinsic parameters when subjects pointed to virtual targets. Data are plotted in the same format as in Fig. 2. 

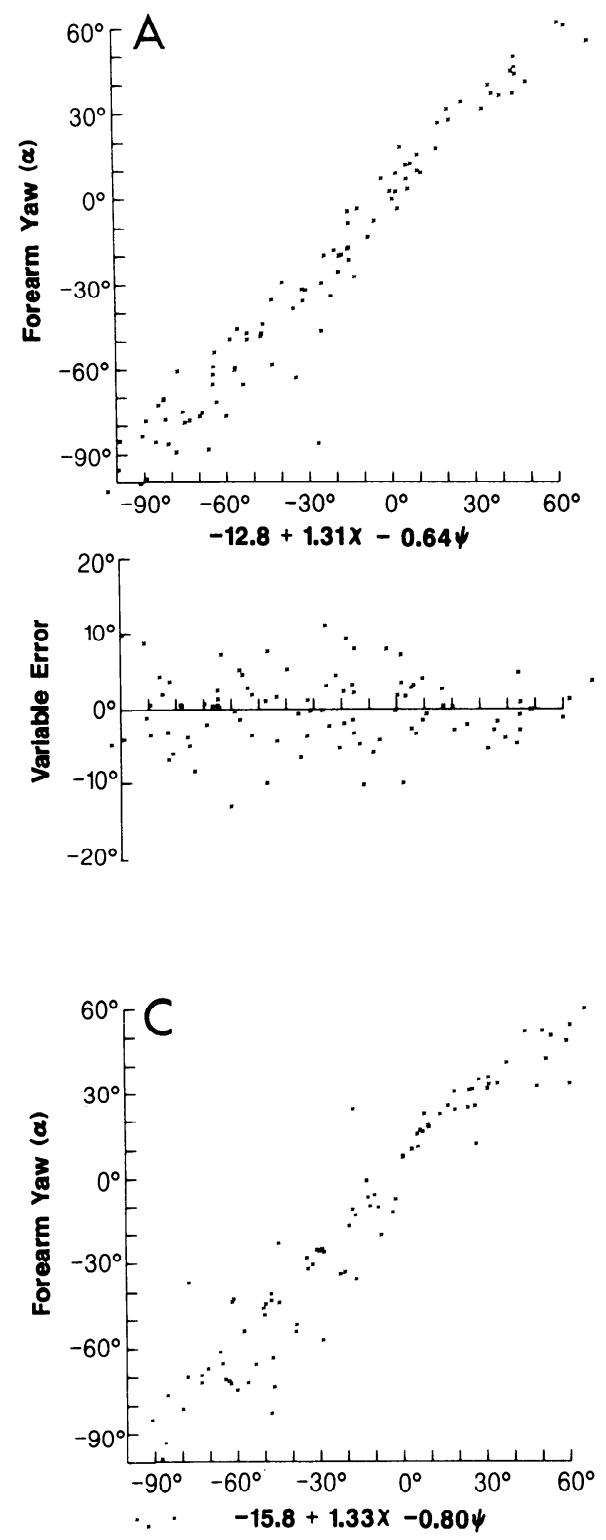
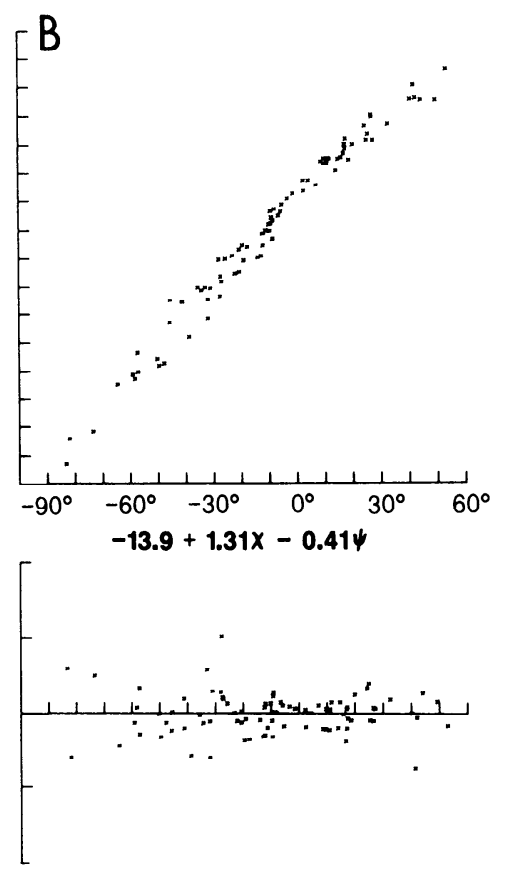

FIG. 7. Dependence of forearm yaw $(\alpha)$ on extrinsic parameters when subjects made accurate movements. Data are plotted in the same format as in Fig. 3.
It is evident on the left (Fig. $4 A$ ) that the sheet defined by the polynomial closely approximates a plane in $R-\psi$ space. There was no significant dependence on target azimuth $(\chi)$. By contrast, on the right, the arm elevation $(\theta)$ did depend significantly on azimuth. The sheets denoted by different line symbols $(-, \cdots,---)$ are plots of the polynomial at constant values of $\chi$, as indicated in the figure. Even at any given value of $\chi$ there was a considerable warping of the surface in $R-\psi$ space, implying the presence of greater nonlinearities in the dependence of $\theta$ on $R$ and $\psi$. This is also apparent in Fig. $4 B$ (top); the data points show much more scatter about a straight line.

Forearm elevation $(\beta)$ also depends primarily on the radial distance to the target $(R)$ and on target elevation $(\psi)$. As is the case for upper arm elevation $(\theta), \beta$ increases with increasing $R$, but it decreases as $\psi$ increases (see Fig. 5 and Table 2). When subjects made errors in pointing to a virtual target in the dark, this dependence of $\beta$ on $R$ and $\psi$ was close to linear (Fig. 5A). When they made accurate movements (Fig. $5 B$ ), there were significant nonlinearities in the dependence of forearm elevation on the three extrinsic parameters defining target location.

Data for the yaw angles (forearm yaw, $\alpha$ and upper arm yaw, $\eta$ ) are shown in Figs. 6-9. Both yaw angles depend significantly on the azimuth $(\chi)$ of target location. To a lesser extent, both of the yaw angles sometimes also depend on radial distance $(R)$ and target elevation $(\psi)$. There was a greater amount of intersubject variability in the dependence of the yaw angles on $R, \chi$, and $\psi$ (Tables 3 and 4). For example, when subjects pointed to a virtual target in the dark, for one subject (Fig. $6 \mathrm{~A}$ ), the linear model showed a significant dependence of forearm yaw $(\alpha)$ only on $\chi$. In one other subject (Fig. $6 B$ ), $\alpha$ depended also on $R$, whereas in the remaining two subjects, $\alpha$ depended on $\chi$ and $\psi$. The extent to which a cubic model improved the fit was variable, yielding only a modest improvement in Fig. 6, $A$ and 

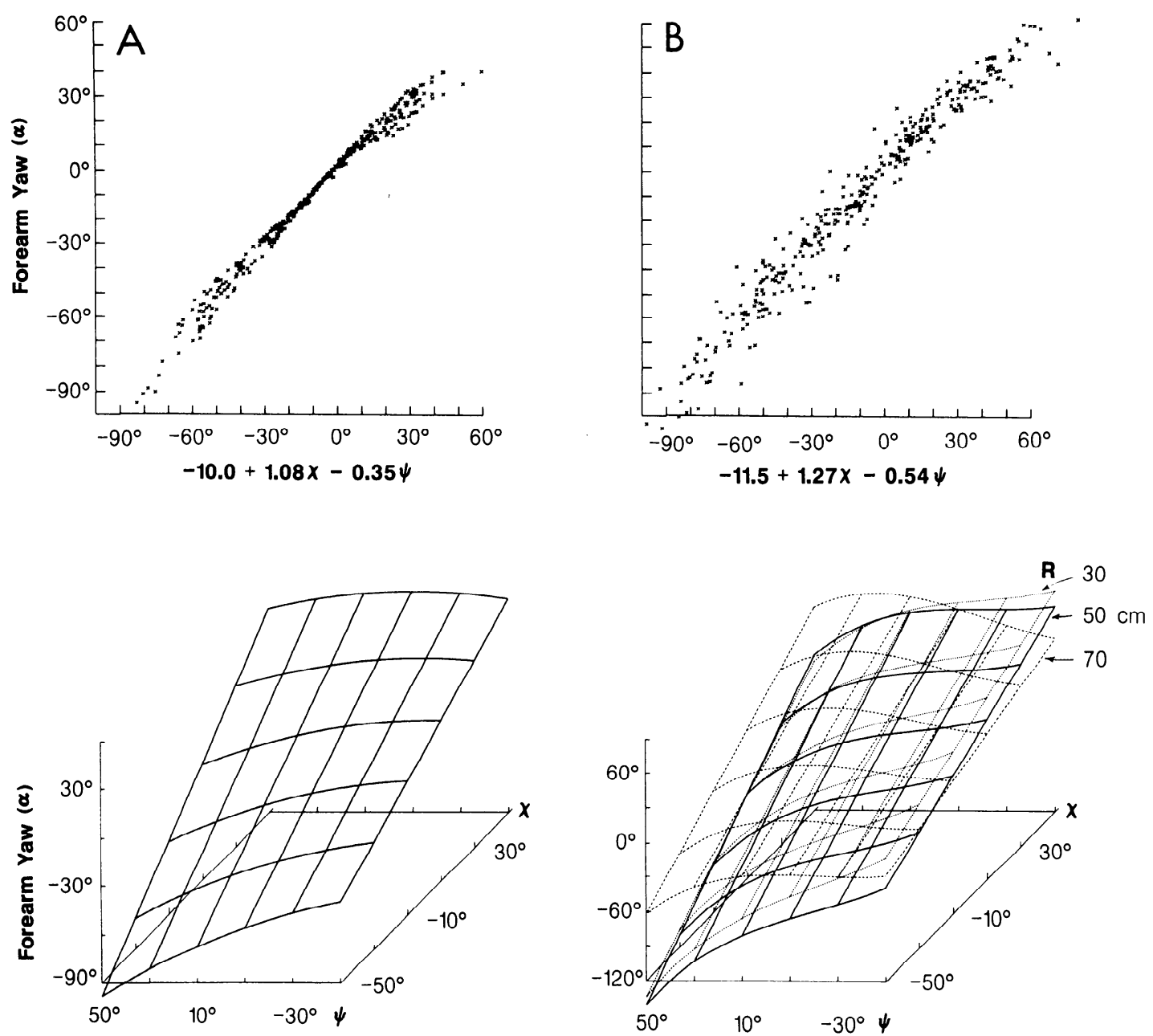

FIG. 8. Relationship between forearm yaw $(\alpha)$ and extrinsic parameters when subjects pointed to virtual targets $(A)$ and when they made accurate movements $(B)$. In $A$ the model did not depend on radial distance $(R)$ although there was such a dependence in $B$.

TABLE 3. Forearm yaw, $\alpha$

\begin{tabular}{|c|c|c|c|c|c|c|}
\hline \multirow[b]{2}{*}{ Subject } & \multirow[b]{2}{*}{ Linear Model } & \multicolumn{2}{|c|}{ Error } & \multirow[b]{2}{*}{$\Delta$} & \multicolumn{2}{|c|}{$r^{2}$} \\
\hline & & $\epsilon_{1}$ & $\epsilon_{\mathrm{c}}$ & & Linear & Cubic \\
\hline \multicolumn{7}{|c|}{ Virtual target (dark) } \\
\hline $\begin{array}{l}A \\
B \\
C \\
D\end{array}$ & $\begin{aligned} 1.5 & +1.02 \chi \\
8.0 & +0.96 \chi-0.21 R \\
-10.1 & +1.22 \chi-0.56 \psi \\
-20.5 & +1.06 \chi-0.62 \psi\end{aligned}$ & $\begin{array}{r}6.18 \\
5.54 \\
7.83 \\
11.86\end{array}$ & $\begin{array}{l}5.84 \\
4.82 \\
5.75 \\
9.26\end{array}$ & $\begin{array}{l}2.03 \\
2.73 \\
5.32 \\
7.41\end{array}$ & $\begin{array}{l}0.947 \\
0.939 \\
0.946 \\
0.883\end{array}$ & $\begin{array}{l}0.953 \\
0.954 \\
0.971 \\
0.929\end{array}$ \\
\hline Combined & $-10.0+1.08 \chi-0.35 \psi$ & 14.77 & 14.26 & 3.86 & 0.795 & 0.809 \\
\hline \multicolumn{7}{|c|}{ Accurate movement } \\
\hline $\begin{array}{l}E \\
F \\
G \\
H\end{array}$ & $\begin{array}{r}-12.8+1.31 \chi-0.64 \psi \\
-13.9+1.31 \chi-0.41 \psi \\
-15.8+1.33 \chi-0.80 \psi \\
-0.2+1.02 \chi-0.19 \psi\end{array}$ & $\begin{array}{r}10.67 \\
5.31 \\
13.55 \\
5.88\end{array}$ & $\begin{array}{l}4.62 \\
2.72 \\
6.21 \\
3.59\end{array}$ & $\begin{array}{r}9.62 \\
4.56 \\
12.04 \\
4.66\end{array}$ & $\begin{array}{l}0.945 \\
0.971 \\
0.916 \\
0.963\end{array}$ & $\begin{array}{l}0.990 \\
0.992 \\
0.982 \\
0.986\end{array}$ \\
\hline Combined & $-11.5+1.27 \chi-0.54 \psi$ & 14.03 & 11.10 & 8.58 & 0.887 & 0.929 \\
\hline
\end{tabular}



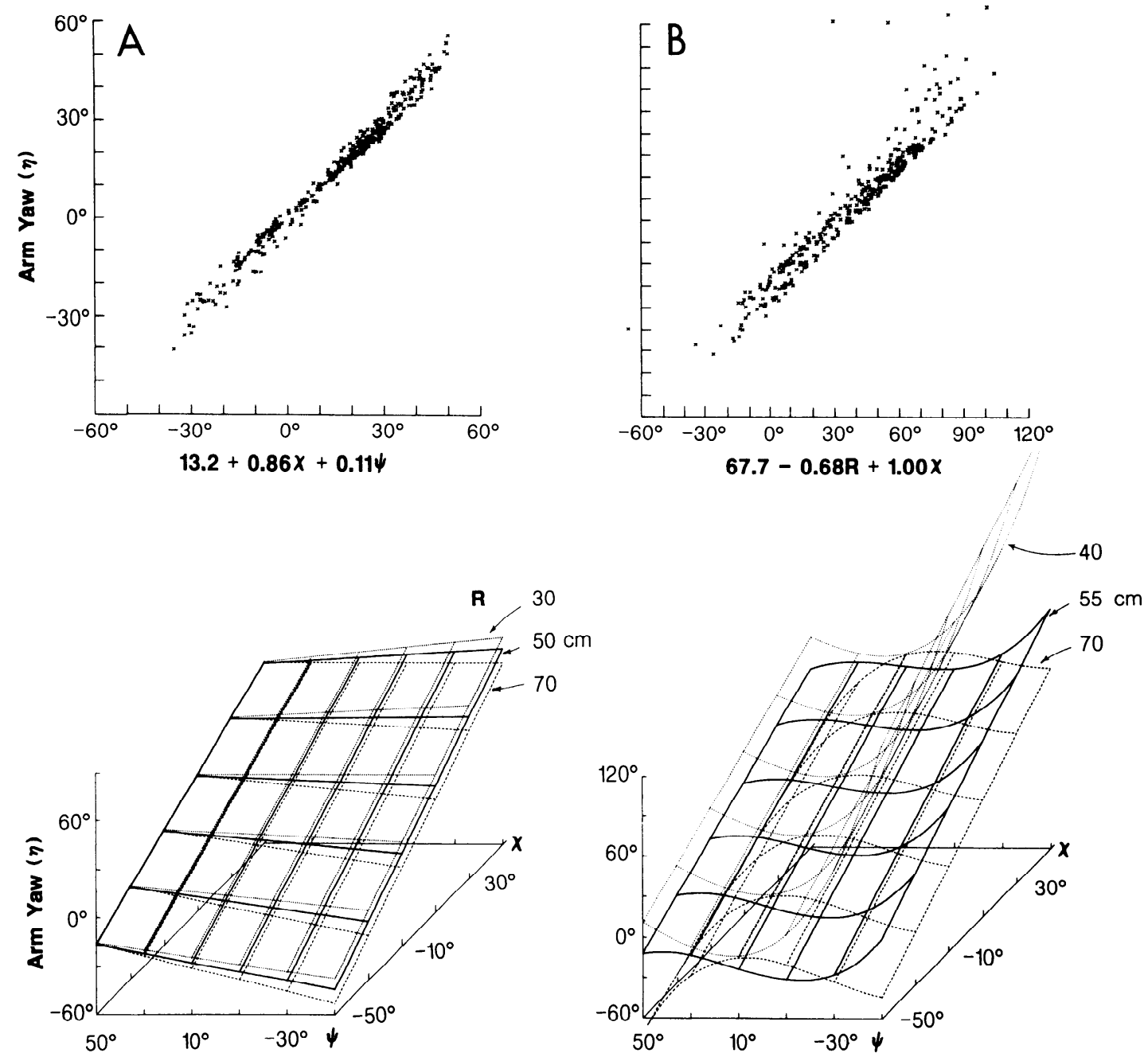

FIG. 9. Relationship between arm yaw $(\eta)$ and extrinsic parameters when subjects pointed to virtual targets $(A)$ and when they made accurate movements $(B)$.

TABLE 4. Upper arm yaw, $\eta$

\begin{tabular}{|c|c|c|c|c|c|c|}
\hline \multirow[b]{2}{*}{ Subject } & \multirow[b]{2}{*}{ Linear Model } & \multicolumn{2}{|c|}{ Error } & \multirow[b]{2}{*}{$\Delta$} & \multicolumn{2}{|c|}{$r^{2}$} \\
\hline & & $\epsilon_{1}$ & $\epsilon_{\mathrm{c}}$ & & Linear & Cubic \\
\hline \multicolumn{7}{|c|}{ Virtual target (dark) } \\
\hline$A$ & $10.7+0.98 \chi+0.26 \psi$ & 12.28 & 11.27 & 4.88 & 0.800 & 0.832 \\
\hline$B$ & $3.2+1.01 \chi+0.16 \psi$ & 8.62 & 7.24 & 4.67 & 0.871 & 0.909 \\
\hline C & $52.6+0.79 \chi-0.56 R$ & 5.68 & 4.42 & 3.58 & 0.914 & 0.948 \\
\hline$D$ & $48.9+0.81 \chi-0.60 R$ & 8.34 & 7.59 & 3.45 & 0.858 & 0.882 \\
\hline Combined & $13.2+0.86 \chi+0.11 \psi$ & 11.56 & 11.36 & 2.15 & 0.757 & 0.765 \\
\hline \multicolumn{7}{|c|}{ Accurate movement } \\
\hline$E$ & $63.6+0.98 \chi-0.53 R$ & 8.76 & 4.28 & 7.64 & 0.927 & 0.983 \\
\hline$F$ & $110.7+0.81 \chi-1.45 R$ & 6.72 & 4.23 & 5.22 & 0.889 & 0.956 \\
\hline$G$ & $72.5+1.08 x-0.97 R$ & 6.80 & 5.54 & 3.94 & 0.956 & 0.971 \\
\hline$H$ & $53.3+0.80 x-0.82 \psi$ & 18.35 & 5.16 & 17.61 & 0.786 & 0.983 \\
\hline Combined & $67.7+1.00 \chi-0.68 R$ & 14.48 & 9.88 & 10.59 & 0.801 & 0.908 \\
\hline
\end{tabular}


$B$ (2.0 and $2.7^{\circ}$, respectively), but giving a much bigger improvement in Fig. $6, C$ and $D\left(5.3\right.$ and $7.4^{\circ}$, respectively).

When subjects made accurate movements to a target, the extent to which a linear model could account for the data was also variable. In two of the four subjects (Fig. 7, $A$ and $C)$ the improvement given by the cubic model ( 9.6 and $\left.12.0^{\circ}\right)$ exceeded that for any of the subjects in Fig. 6 . The results for the other two subjects $\left(4.6\right.$ and $\left.4.7^{\circ}\right)$ were intermediate to the extremes found when subjects made movements which could be inaccurate (Fig. 6).

Pooled data from all four subjects for the two experimental conditions are shown in Fig. 8. Once again it is clear that the dependence of forearm yaw $(\alpha)$ on the target parameters is much closer to linearity when subjects pointed to a virtual target in the dark (Fig. $8 A$ ) than when they made accurate movements (Fig. $8 B$ ).

A similar conclusion holds true for upper arm yaw $(\eta)$, as may be seen in Fig. 9. Data for individual subjects are reported in Table $4 .^{1}$ In three of the four subjects who made accurate movements, the improvement in fit $(\Delta)$ given by the cubic model exceeded that for any of the subjects who made inaccurate movements. The value for the fourth subject was bracketed by the values for the four subjects in the first task.

To summarize, when subjects pointed to a virtual target in the dark each of the four intrinsic parameters which describe the orientation of the arm and forearm $(\eta, \theta, \alpha, \beta)$ depended in a close to linear fashion on one or two of the extrinsic parameters which describe the spatial location of the target $(R, \chi$, and $\psi)$. The two elevation angles $(\theta$ and $\beta)$ depend on target distance $(R)$ and elevation $(\psi)$, whereas the two yaw angles $(\eta$ and $\alpha$ ) depend primarily on target azimuth $(\chi)$ with a weaker dependence on $\psi$. When subjects made accurate movements to a target, these relationships between intrinsic and extrinsic coordinates became significantly more nonlinear.

This conclusion is supported by the combined data from all four subjects in each experimental condition (Figs. 4, 5, 8 , and 9 , and Tables 1-4). For each of the four orientation angles, we did an analysis of variance to compare $\Delta$ (the amount by which a nonlinear model gives a better fit) between the two experimental conditions (see Tables 1-4). For all four angles, $\Delta$ was significantly larger for the data from accurate movements, at the 0.01 level. When results from individual subjects are examined, there was some overlap between the two experimental conditions. Nevertheless, for all four orientation angles, the smallest improvement in the nonlinear model over the linear model was found in the first experimental condition (pointing to a virtual target) and the largest in a subject in the second experimental condition.

\section{DISCUSSION}

The starting point of the analysis presented in this paper is that the spatial location of a target and the orientation of

\footnotetext{
${ }^{1}$ When the angular elevation of the shoulder is small, the uncertainty in estimating the yaw angle of the $\operatorname{arm}(\eta)$ becomes large. Therefore we neglected from the analysis those trials in which $\theta$ was $<15^{\circ}$.
}

the arm are represented in different coordinate systems. We call the parameters which denote target location extrinsic coordinates and those which denote limb orientation intrinsic coordinates. Note that both of these coordinate systems can be used to define the same point in space. (When a subject points to an extrinsic target, the position of the finger tip can be derived from the intrinsic coordinates.) In fact, the execution of a pointing task can be thought to involve a mapping between these two coordinate systems.

Departing from this assumption, we examined the relationship between these intrinsic and extrinsic variables under two experimental conditions: 1) pointing to a virtual target in the dark and 2) pointing to a target which was physically present and both it and the arm were visible to the subject. In the preceding paper (Soechting and Flanders 1989) we showed that subjects could miss the virtual target by as much as $15 \mathrm{~cm}$. In this paper we showed that the relationship between intrinsic and extrinsic coordinates was close to linear when subjects made inaccurate movements to virtual targets (first experimental condition). We also showed that the dependence of each of the intrinsic coordinates on the extrinsic parameters was much more nonlinear when they made accurate movements (second experimental condition). From these observations we derive the following hypotheses: movement inaccuracy results because the subjects map the target location into the space of intrinsic parameters and because this mapping involves quasi-linear approximations. Accurate movements invoke corrections which introduce substantial nonlinearities into the relationship between intrinsic and extrinsic parameters.

As for the details of this mapping, both the elevation of the $\operatorname{arm}(\theta)$ and of the forearm $(\beta)$ are related linearly to target distance from the shoulder $(R)$ and the elevation of the target above or below the shoulder $(\psi)$. The two yaw angles $(\eta$ and $\alpha$ ) are related linearly to the target azimuth $(\chi)$. The two yaw angles depend also to a lesser extent on target distance and elevation in a manner which appears to be idiosyncratic to each subject. In addition, when subjects make inaccurate movements, there are small but variable nonlinearities which differ from subject to subject. In the APPENDIX we derive analytically the conditions under which such linear approximations are most appropriate for attaining the target.

Our hypotheses are strengthened by results that we have presented previously. First, the intrinsic and extrinsic coordinate systems were not chosen ad hoc. Our choice of intrinsic coordinates was based on psychophysical studies (Soechting et al. 1982, 1984; Worringham et al. 1987) in which we showed that subjects were best able to match each of the orientation angles of their right and left arms when these angles were represented in the coordinate system used here. Our rationale for using spherical coordinates centered at the shoulder has been given in the preceding paper (Soechting and Flanders 1989).

Second, our hypothesis that movement error results from linear approximations between intrinsic and extrinsic parameters gains support from kinematic studies of drawing movements (Soechting et al. 1986a,b). In those studies we asked subjects to draw circles and ellipses in different 
planes in three-dimensional space. As in the present study, we found that the trajectory of the wrist in space could show considerable distortion. We also found linear relations between variables which describe the motion in extrinsic and intrinsic coordinate frames. Specifically we found that the phase difference in the modulation in the two yaw angles was linearly related to the azimuth of the plane in which the ellipse was drawn. Furthermore, the phase between forearm yaw and elevation angles was linearly related to the slant of the ellipse. Simulation studies showed that the observed distortions of the trajectory could be predicted on the basis of a linear mapping between extrinsic and intrinsic coordinates. We also showed that more complicated continuous movements (such as figure eights and stars drawn in space) are segmented, each segment constituting an arc of an ellipse generated according to the linear transformations just described (Soechting and Terzuolo 1987a,b).

Thus there is experimental evidence that is consistent with the hypothesis that there exist linear mappings between extrinsic and intrinsic coordinates both for continuous as well as for point-to-point movements. Furthermore such linear mappings can account for the observed movement errors. Therefore, the assumption that coordinated movement involves sensorimotor transformations between different kinematic representations (intrinsic and extrinsic) and the hypothesis that these transformations are accomplished by means of linear approximations can account for the performance of a large variety of motor tasks.

The sensorimotor transformation which we have hypothesized and which we have discussed above would be but one stage in the information processing which occurs between the presentation of a target and the issuance of the appropriate motor commands to the muscles. In the following, we will discuss other possible stages in this process in the light of available experimental evidence.

The location of a target is initially represented in a retinotopic frame of reference. The extrinsic frame of reference in which we have chosen to represent the target is a body-centered one, with the origin at the shoulder. Thus an initial transformation would involve a mapping of visually derived information from a retinotopic to a body-centered frame of reference. There is considerable evidence in favor of such a mapping, both for the control of eye movements (Mays and Sparks 1980; Robinson 1975; Sparks 1988) as well as for limb movements (Andersen 1987). One area which has been implicated in this transformation is posterior parietal cortex (cf. Andersen 1987); lesions of this area produce errors in visually guided reaching. Recently, Andersen and Zipser (1988) recorded from neurons in a subdivision of this region (area 7a) and found neurons whose responses depend on both the retinal location of the stimulus and the position of the eyes in the head. Thus these neurons may participate in the transformation from a retinotopic frame of reference to a body-centered frame of reference.

Once target location is represented in body-centered coordinates, arm movement to a target could be achieved in principle by means of one transformation: mapping from target location to an appropriate level of activation of each of the limb muscles. In its original formulation, the equilibrium point hypothesis (Kelso and Holt 1980; Polit and Bizzi 1979) adopted such a scheme: to each point in space corresponds a given length of each of the muscles of the limb. The appropriate posture of the limb could be achieved by taking advantage of the spring-like properties of muscle and by specifying the appropriate equilibrium point for each of the muscles.

The hypothesis was attractive since the initial position of the limb need not be known to set the equilibrium point. However, evidence has accumulated against this hypothesis (Day and Marsden 1982; Hasan and Enoka 1985; Sanes and Evarts 1983) and the data indicate that, from the perspective of the equilibrium-point hypothesis, this point shifts gradually from the initial posture of the limb to its final posture (Bizzi et al. 1984; Flash 1987; Hogan 1985). Thus both the initial posture and the final posture are used to specify the appropriate patten of muscle activation.

Since information about limb posture can be kinesthetically derived (accurate and direct limb movements can be made even when the limb is not in view at the onset of the movement), information about initial limb position and target location are subserved by two different sensory modalities. One way to utilize information about initial limb posture and target location is to transform the latter representation into the former.

The data presented in this paper suggest how this might be accomplished. The orientation angles of the arm would be used to represent both the initial location of the finger as well as the desired final location of the finger. The desired final location of the finger (at the target) would be derived by means of the transformation described in this paper. Once both the initial and final positions are represented in terms of joint angles, it is possible to derive the direction and amplitude of the movement required to attain the target by taking the vectorial difference. (Note that this hypothesis does not require that the final joint angles be encoded explicitly in the activity of some population of neurons. The vectorial difference could in principle be derived without the benefit of such an intermediate representation by the appropriate (linear) combination of neural activities encoding target location in a body-centered frame of reference and initial limb position.)

There is evidence that movement direction is indeed represented in the discharge of cortical neurons, independently of the spatial locus of target or hand. In particular Georgopoulos and colleagues (1982) and Schwartz et al. (1988) have shown that neurons in motor cortex and in area 5 exhibit such directional tuning, the firing frequency of a given neuron varying with the cosine of the angle between the neuron's preferred direction and the direction of the required movement. They have also shown that movement direction is predicted by the population discharge of these neurons, each having its own preferred clirection (Georgopoulos et al. 1983, 1988; Kettner et al. 1988). How and where movement amplitude might be represented is not known. Neither do we know how and where appropriate levels of muscle activation might be derived from a neural representation of direction and amplitude (kinematics), although some speculations on this topic 
have been advanced (Georgopoulos et al. 1988; Schwartz et al. 1988).

The hypothesis we have outlined implies that there is an explicit kinematic representation of movement direction and amplitude and that the errors in pointing arise in the process of computing these kinematic quantities. While our data are consistent with this hypothesis, the possibility exists that the kinematic representation is accurate and that movement errors are due to approximations in computing torques and/or muscle activation. This alternate possibility involves a direct transformation from the representations of initial limb posture and target location to muscle force. It is not clear why a direct transformation would produce the type of movement errors reported here.

One final point. The transformations we have described in this paper represent mathematical simplicity. It must be acknowledged that mathematical simplicity does not necessarily imply simplicity in implementing such rules in neural network structures. Until more is known about the neural substrates of the behavior we have described, it is also difficult to say much on this point. However, in other sensorimotor systems linear transformations have proven to be useful descriptions of neural activity (Baker et al. 1984; Gielen and van Zuylen 1986; Robinson 1982, 1985; Simpson and Graf 1985).

\section{APPENDIX}

In this APPENDIX we explore analytically how and what linear approximations between intrinsic and extrinsic variables may be used to arrive in the proximity of the target.

In a spherical coordinate system

$$
\begin{aligned}
R^{2} & =x^{2}+y^{2}+z^{2} \\
\tan \psi & =z / \sqrt{\left(x^{2}+y^{2}\right)} \\
\tan \chi & =x / y
\end{aligned}
$$

and making use of $E q . l$ and the assumption that the lengths $l$ of the arm and forearm are equal, then

$$
\begin{aligned}
R^{2} & =2 l^{2}(1-\cos \theta \cos \beta+2 \sin \theta \sin \beta \cos \gamma) \\
\tan \psi & =(\cos \beta-\cos \theta) /\left(\sin ^{2} \theta+\sin ^{2} \beta+2 \sin \theta \sin \beta \cos \gamma\right) \\
\tan \chi & =(\sin \theta \sin \eta+\sin \beta \sin \alpha) /(\sin \theta \cos \eta+\sin \beta \cos \alpha)
\end{aligned}
$$

where

$$
\gamma=\eta-\alpha
$$

If $\gamma$ is small, i.e., if the two yaw angles $\eta$ and $\alpha$ are approximately equal, one can make the small angle approximation

$$
\cos \gamma \simeq 1-\gamma^{2} / 2
$$

to obtain

$$
\left.R=2 l \sin \frac{(\theta+\beta)}{2}\left\{1-\frac{\gamma^{2}}{4} \sin \theta \sin \beta / \sin ^{2} \frac{(\theta+\beta)}{2}\right)+\cdots\right\}
$$

$\tan \psi=-\tan \frac{(\beta-\theta)}{2}$

$$
\times\left\{1+\frac{\gamma^{2}}{2} \sin \theta \sin \beta /(\sin \theta+\sin \beta)^{2}+\cdots\right\}
$$

and

$\tan \chi=\tan \eta\{1-\gamma(\sin \beta \cos \eta+\sin \beta \sin \eta) / \sin \theta+\cdots\}$
From $E q . A 5$ it is clear that if $\eta$ and $\alpha$ are approximately equal, $\gamma$ is negligible and

$$
\eta=\alpha=\chi
$$

Thus one might expect both yaw angles to be linearly related to target azimuth with a slope of unity. Experimentally this is close to the value actually found. However, both yaw angles also depended on target elevation, and this is not predicted by this simple analysis.

If one neglects $\gamma$ in Eqs. $A 3$ and $A 4$, one obtains

$$
\begin{aligned}
& R=2 l \sin \frac{(\theta+\beta)}{2} \\
& \psi=(\theta-\beta) / 2
\end{aligned}
$$

and making the additional assumption that $\theta+\beta$ is small

$$
R / l=(\theta+\beta)
$$

Solving Eqs. $A 8$ and $A 9$ for $\theta$ and $\beta$, one obtains (assuming $l \simeq 30$ $\mathrm{cm}$ )

$$
\begin{aligned}
& \beta=R-\psi \\
& \theta=R+\psi
\end{aligned}
$$

where the angles are expressed in degrees and $R$ in centimeters.

The expression for $\theta(E q . A 10)$ is close to the experimental data reported in Table 1 . In Table 2 , the regression coefficient for $\psi$ is also close to unity; however, that for $R$ is closer to 0.5 . Thus some of the data reported in Tables $1-4$ is predicted by the linear approximations developed in this APPENDIX, others are not.

The question remains: is such a linear approximation warranted? The small angle approximation for $\theta+\beta$ is certainly suspect; experimentally, $\theta$ and $\beta$ ranged from 0 to $\geq 90^{\circ}$. The assumption that the two yaw angles $\eta$ and $\alpha$ are approximately equal was valid for some subjects and not for others. For example among the subjects who pointed to a virtual target in the dark, $\eta$ and $\alpha$ tended to covary for subjects $A$ and $B$. For these subjects the slope of the regression of $\eta$ on $\alpha$ was 0.92 and 0.93 , respectively, with correlation coefficients $r^{2}$ equal to 0.806 and 0.763 , respectively. For the other two subjects ( $C$ and $D$ ), this was not the case, the slope of the regression being 0.47 for both, with $r^{2}$ equal to 0.664 and 0.555 , respectively.

Thus it is clear that the linearization we have outlined will not uniformly lead to movement accuracy. Nevertheless, such a procedure may be useful in obtaining a ballpark estimate (Greene 1972) which could be further improved by empirically adding nonlinear corrections. The data we have presented in this paper suggest that, in fact, this is what our subjects tended to do.

This work was supported by National Institute of Neurological and Communicative Disorders and Stroke Grant NS-15018 and the National Science Foundation Grant BNS-8418539.

Address for reprint requests: J. F. Soechting, Dept. of Physiology, University of Minnesota, Minneapolis, MN 55455.

Received 9 November 1988; accepted in final form 3 April 1989.

\section{REFERENCES}

ANDERSEN, R. A. Inferior parietal lobe function in spatial perception and visuomotor integration. In: Handbook of Physiology. The Nervous System. Higher Functions of the Brain. Bethesda, MD: Am. Physiol. Soc., 1987, sect. 1, vol. V, pt 2, chapt. 12, p. 483-518.

ANDERSEN, R. A. AND ZIPSER, D. The role of posterior parietal cortex in coordinate transformations for visual-motor integration. Can. J. Physiol. Pharmacol. 66: 488-501, 1988.

Baker, J., Goldberg, J., Herrmann, G., and Peterson, B. Optimal response planes and canal convergence in secondary neurons in vestibular nuclei of alert cats. Brain Res. 294: 133-137, 1984. 
Bizzi, E., ACcornero, N., Chapple, W., AND Hogan, N. Posture control and trajectory formation during arm movement. J. Neurosci. 4: 2738-2744, 1984.

DAY, B. L. AND MARSDEN, C. D. Accurate positioning of the human thumb against unpredictable dynamic loads is dependent upon peripheral feedback. J. Physiol. Lond. 327: 393-408, 1982.

FLASH, T. The control of hand equilibrium trajectories in multi-joint arm movements. Biol. Cybern. 57: 257-274, 1987.

Georgopoulos, A. P., Caminiti, R., Kalaska, J. F., and Massey, J. T Spatial coding of movement: a hypothesis concerning the coding of movement direction by motor cortical populations. Exp. Brain Res. 7, Suppl.: 327-336, 1983.

Georgopoulos, A. P., Kalaska, J. F., CAMINiti, R., AND MasSey, J. T. On the relations between the direction of two-dimensional arm movements and cell discharge in primate motor cortex. J. Neurosci. 2 : $1527-1537,1982$.

Georgopoulos, A. P., Kettiner, R. E., And Schwartz, A. B. Primate motor cortex and free arm movements to visual targets in three-dimensional space. II. Coding of the direction by a neuronal population. $J$. Neurosci. 8: 2928-2937, 1988.

GiELEN, C. C. A. M. AND VAN ZUYLEN, E. J. Coordination of arm muscles during flexion and supination: application of tensor analysis approach. Neuroscience 17: 527-539, 1986.

Goodwin, G. M., MCCloskey, D. I., and Matthews, P. B. C. The contribution of muscle afferents to kinaesthesia shown by vibration induced illusions of movements and by the effects of paralysing joint afferents. Brain 95: 705-748, 1972.

GREENE, P. H. Problems of organization of motor systems. Progr. Theor. Biol. 2: 304-338, 1972

HASAN, Z. AND ENOKA, R. M. Isometric torque-angle relationships and movement-related activity of human elbow flexors: implications for the equilibrium point hypothesis. Exp. Brain Res. 59: 441-450, 1985.

HoGAN, N. The mechanics of multi-joint posture and movement control. Biol. Cybern. 52: 315-332, 1985.

Horch, K. W., Clark, F. J., and Burgess, P. R. Awareness of knee joint angle under static conditions. J. Neurophysiol. 38: 1436-1447, 1975.

Johnson, R. A. AND WiChern, D. W. Applied Multivariate Statistical Analysis. Englewood Cliffs, NJ: Prentice Hall, 1982, p. 291-358.

KELSO, J. A. S. AND HOLT, K. G. Exploring a vibratory system analysis of human movement production. J. Neurophysiol. 43: 1183-1196, 1980.

Kettner, R. E., Schwartz, A. B., AND Georgopoulos, A. P. Primate motor cortex and free arm movements to visual targets in three-dimensional space. III. Positional gradients and population coding of movement direction from various movement origins. J. Neurosci. 8: 2938-2947, 1988.

LASZLO, J. I. AND BAIRsTow, P. J. Kinaesthesis: its measurement, training and relationship to motor control. Q. J. Exp. Psychol. 35A: 411-421, 1983.

MAYS, L. E. AND SPARKS, D. L. Dissociation of visual and saccade-related responses in superior colliculus neurons. J. Neurophysiol. 43: 207-232, 1980.
NASHNER, L. M. AND MCCOLluM, G. The organization of human postural movements: a formal basis and experimental synthesis. Behav. Brain Sci. 8: 135-172, 1985.

PAILlARD, J. Muscular proprioception and position sense. Arch. Ital. Biol. 111: 451-461, 1973.

POLIT, A. AND BIZZI, E. Characteristics of motor programs underlying arm movements. J. Neurophysiol. 42: 183-194, 1979.

RoBINSON, D. A. Oculomotor control signals. In: Basic Mechanisms of Ocular Motility and their Clinical Implications, edited by P. Bach-yRita and G. Lennerstrand. London: Pergamon, 1975, p. 337-374.

RoBINSON, D. A. The use of matrices in analyzing the three-dimensional behavior of the vestibulo-ocular reflex. Biol. Cybern. 46: 53-66, 1982.

RoBINSON, D. A. The coordinates of neurons in the vestibulo-ocular reflex. Rev. Oculomot. Res. 1: 297-312, 1985.

SANES, J. N. AND EvARTS, E. V. Effects of perturbations on accuracy of movements. J. Neurosci. 3: 977-986, 1983.

Schwartz, A. B., Kettner, R. E., AND Georgopoulos, A. P. Primate motor cortex and free arm movements to visual targets in three-dimensional space. I. Relations between single cell discharge and direction of movement. J. Neurosci. 8: 2913-2927, 1988.

SIMPSON, J. I. AND GRAF, W. The selection of reference frames by nature and its investigation. Rev. Oculomot. Res. 1: 3-20, 1985.

SOECHTING, J. F. Does position sense at the elbow reflect a sense of elbow joint angle or one of limb orientation? Brain Res. 248: 392-395, 1982.

SOECHTING, J. F. AND FlANDERS, M. Sensorimotor representations for pointing to targets in three-dimensional space. J. Neurophysiol. 62: 582-594, 1989.

Soechting, J. F., LaCQuaniti, F., AND Terzuolo, C. A. Coordination of arm movements in three-dimensional space. Sensorimotor mapping during drawing movement. Neuroscience 17: 295-311, 1986a.

SOECHTING, J. F. AND Ross, B. Psychophysical determination of coordinate representation of human arm orientation. Neuroscience 13: 595-604, 1984.

SOECHTING, J. F. AND TERZUOLO, C. A. An algorithm for the generation of curvilinear wrist motion in an arbitrary plane in three-dimensional space. Neuroscience 19: 1395-1405, 1986b.

SoEChting, J. F. AND TeRzUOLO, C. A. Organization of arm movements. Motion is segmented. Neuroscience 23: 39-52, 1987a.

SOECHTING, J. F. AND TERZUOLO, C. A. Organization of arm movements in three-dimensional space. Wrist motion is piece-wise planar. Neuroscience 23: 53-61, $1987 \mathrm{~b}$.

SPARKS, D. L. Translation of sensory signals into commands for control of saccadic eye movements: role of primate superior colliculus. Physiol. Rev. 66: 118-171, 1986.

SPARKS, D. L. Saccadic command signals in the superior colliculus: implications for sensorimotor transformations. Can. J. Physiol. Pharmacol. 66: 527-531, 1988.

SPARKS, D. L. AND Nelson, J. S. Sensory and motor maps in the mammalian superior colliculus. Trends Neurosci. 10: 312-317, 1987.

Worringham, C. J., STElmaCh, G. E., AND Martin, Z. E. Limb segment inclination sense in proprioception. Exp. Brain Res. 66: 653-658, 1987. 